\title{
Sports related to drowning
}

\author{
David Szpilman ${ }^{1}$ and James P. Orlowski ${ }^{2,3}$
}

\author{
Number 2 in the Series "Sports-related lung disease" \\ Edited by Yochai Adir and Alfred A. Bove
}

\begin{abstract}
Affiliations: 'Sociedade Brasileira de Salvamento Aquatico - SOBRASA, Rio de Janeiro, Brazil. ${ }^{2}$ Division of Pediatrics, Dept of Pediatric Critical Care Medicine, Florida Hospital Tampa, Tampa, FL, USA. ${ }^{3}$ Dept of Pediatrics and Critical Care Medicine, Johns Hopkins All Childrens Hospital, St Petersburg, FL, USA.
\end{abstract}

Correspondence: David Szpilman, Av. das Américas 3555, bloco 2, sala 302, Barra da Tijuca, Rio de Janeiro, 22631-003, Brazil. E-mail: davidaszpilman.com

ABSTRACT Aquatic sports are included in the top list of risky practices as the environment per se carries a possibility of death by drowning if not rescued in time. Not only are aquatic sports related to a high risk of death, but also all sports practiced on the water, over the water and on ice. Whatever the reason a person is in the water, drowning carries a higher possibility of death if the individual is unable to cope with the water situation, which may simply be caused by an inability to stay afloat and get out of the water or by an injury or disease that may lead to physical inability or unconsciousness. The competitive nature of sports is a common pathway that leads the sports person to exceed their ability to cope with the environment or simply misjudge their physical capability. Drowning involves some principles and medical interventions that are rarely found in other medical situations as it occurs in a deceptively hostile environment that may not seem dangerous. Therefore, it is essential that health professionals are aware of the complete sequence of action in drowning. This article focuses on the pulmonary injury in sports and recreational activities where drowning plays the major role.

$@$ ERSpublications

Drowning involves rarely seen interventions: it is essential health professionals are aware of the drowning sequence http://ow.ly/yjUz3009Qup

\section{Introduction}

Worldwide, more and more people are participating in sports to have fun, to exercise, for competition or health purposes. Aquatic sports are included in the top list of risky practices, as the environment per se carries a possibility of death by drowning if the individual is not rescued in time. Not only are the aquatic sports related to a high risk of death, but so are all sports practised on water (e.g. sailing), over water (e.g. hang-gliding) and on ice (skiing or skating). Table 1 describes sports practised in, on, near, under, over and in ice or water, which may lead to drowning.

Whatever the reason for being in the water, there is a higher possibility of drowning leading to death if the individual is unable to cope with the water situation, which may be caused by an inability to stay afloat and get out of the water or by an injury or disease that may lead to physical inability or unconsciousness [1]. The competitive nature of sports is a common pathway that leads the sportsperson to exceed their ability to cope with the environment or simply misjudge their physical capability.

Previous articles in this series: No. 1: Adir Y, Bove AA. Can asthmatic subjects dive? Eur Respir Rev 2016; 25: $214-220$.

Received: April 212016 | Accepted after revision: May 052016

Conflict of interest: None declared.

Provenance: Submitted article, peer reviewed.

Copyright CERS 2016. ERR articles are open access and distributed under the terms of the Creative Commons Attribution Non-Commercial Licence 4.0. 
TABLE 1 Sports undertaken in, on, near, under, over and in water or ice, which may lead to drowning

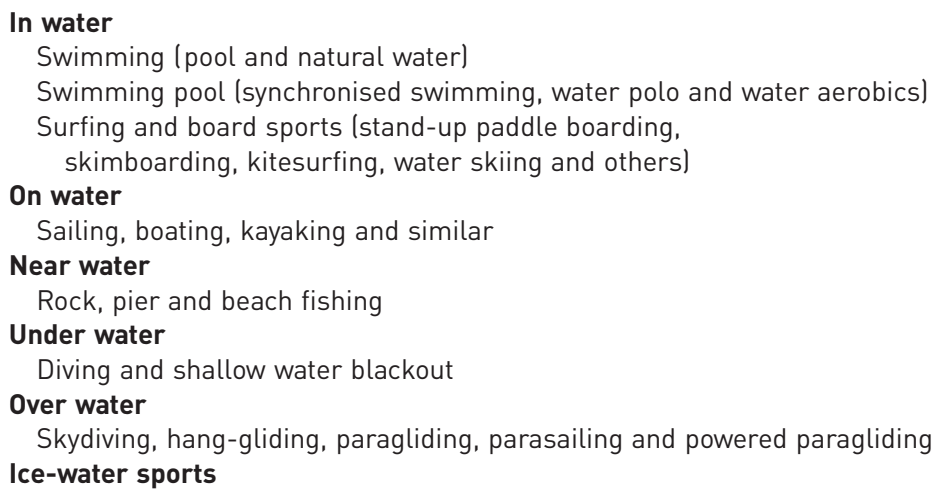

According to the World Health Organization [2] drowning is a serious and neglected public health threat claiming the lives of 372000 people a year worldwide. Fatal drowning is a leading cause of death worldwide among males aged 5-14 years. Over $90 \%$ of these deaths occur in low- and middle-income countries. The daily toll of this leading global killer continues its quiet rise [1].

International data severely underestimate the actual drowning figures, even for high-income countries [3]. Epidemiological data account for only a small part of the problem, because drowning is only partially or not registered in almost all countries. Drowning deaths due to disasters are not included and death certificates usually register the complication of drowning as the cause of death, not the incidence of drowning itself. Almost all drowning victims are able to help themselves or are rescued in time by bystanders or professional rescuers and are not registered at all. In areas where lifeguard services operate fully [1], $<1 \%$ of all persons rescued by lifeguards needed cardiopulmonary resuscitation (CPR) and $0.34 \%$ of the rescues resulted in the death of the victim [4]. In one report of rescues by bystanders, almost $30 \%$ of persons rescued from drowning required CPR [5], which demonstrates that a delay in intervention may lead to a more severe drowning picture than if lifeguards are on duty. The bias produced by not accounting for the real drowning figures gives the false impression that every drowning requires CPR and that to know how to resuscitate is the most important tool to save people from drowning [6]. For every person who dies from drowning, at least another four receive care in the emergency department for nonfatal drowning [1].

Exposure-adjusted, person-time estimates for drowning are 200 times higher than such estimates for deaths from traffic accidents [7]. Key risk factors for drowning are male sex, age <14 years, aquatic exposure, risky behaviour, lack of supervision, alcohol use, low income, poor education and rural residency (the former five factors relate to aquatic sports). For people with epilepsy, the risk of drowning related to aquatic sports is 15-19 times higher than the risk for those who do not have epilepsy [1].

Drowning involves some physiological principles and medical interventions that are rarely found in other medical situations, as it occurs in a deceptively hostile environment that may not seem dangerous. Therefore, it is essential that health professionals are aware of the complete sequence of steps towards survival in drowning [6].

This article focuses on the pulmonary injury in sports and recreational activities where drowning plays the major role.

\section{Definition of drowning}

Drowning is "the process of experiencing respiratory impairment from submersion or immersion in liquid" [8]. This process is a continuum, beginning with respiratory impairment as the victim's airway goes below the surface of the liquid (submersion) or when water splashes over the face (immersion). If the victim is rescued at any time, the process of drowning is interrupted: a nonfatal drowning. If the victim dies at any time this is a fatal drowning. Any submersion or immersion incident without evidence of respiratory impairment (aspiration) should be considered a water rescue and not a drowning. Terms such as "near-drowning", "dry or wet drowning", "active and passive drowning" and "delayed onset of respiratory distress" should not be used $[8,9]$.

\section{Pathophysiology of drowning}

When a drowning person can no longer keep the airway clear, the water that enters the mouth is voluntarily spat out or swallowed. When water is aspirated into the airways, coughing occurs as an initial reflex 
response. In $<2 \%$ of cases $[10,11]$ laryngospasm may be present when the victim starts to inhale water. If the person is not rescued, aspiration of water continues and hypoxaemia leads to loss of consciousness and apnoea in the seconds to a few minutes preceding cardiac arrest. As a consequence, hypoxic cardiac arrest generally occurs after a period of bradycardia and pulseless electrical activity, and not by means of ventricular fibrillation $[12,13]$. The whole drowning process, from submersion or immersion to cardiac arrest, usually occurs within seconds to a few minutes, but in unusual situations, such as rapid hypothermia, this process can last for up to an hour [14]. In most incidents of drowning the heart tissue is relatively healthy and ceases perfusion due to hypoxic insult after a period of apnoea $[4,15]$. If the person is rescued in time during this process the clinical picture is determined by the reactivity of the airways and the amount of water that has been aspirated, but not the type of water (salt or fresh). Water in the alveoli causes surfactant destruction and wash-out. Salt and fresh water aspiration cause similar pathology [12]. In either situation, the effect of the osmotic gradient on the alveolar-capillary membrane can disrupt the integrity of the membrane, increase its permeability and exacerbate fluid, plasma and electrolyte shifts [12]. The clinical picture of the damage is a regional or generalised pulmonary oedema, which may alter the exchange of oxygen in varying proportions $[4,12,16]$. In animal research [16], the aspiration of $2.2 \mathrm{~mL} \cdot \mathrm{kg}^{-1} \mathrm{water}$ led to a severe disturbance to oxygen exchange, decreasing the arterial oxygen pressure $\left(\mathrm{PaO}_{2}\right)$ to $\sim 60 \mathrm{mmHg}$ within $3 \mathrm{~min}$. In humans, it seems that as little as $1-3 \mathrm{~mL} \cdot \mathrm{kg}^{-1}$ of water aspiration produces profound alterations in pulmonary gas exchange and decreases pulmonary compliance by $10-40 \%$ [12]. The combined effects of fluid in the lungs, loss of surfactant and increased capillary-alveolar permeability can result in decreased lung compliance, increased right-to-left shunt in the lungs, atelectasis and alveolitis [12].

One of the unique features, and a last stage of the drowning, is that apnoea and hypoxia cause and precede the cardiac arrest by a few seconds/minutes. In most cases of drowning the heart tissue is relatively healthy and stops due to the hypoxia insult, after a period of apnoea $[4,15]$. If the victim needs CPR, neurological damage is similar to other cardiac arrest situations, but there are exceptions. Hypothermia associated with drowning can provide a protective mechanism that allows victims to survive prolonged submersion episodes $[1,14]$. Hypothermia can reduce brain oxygen consumption, prolonging the interval until cellular anoxia and ATP depletion occur. Hypothermia reduces the electrical and metabolic activity of the brain in a temperature dependent fashion. The rate of cerebral oxygen consumption is reduced by $\sim 5 \%$ per degree Celsius reduction in temperature within the range of $20-37^{\circ} \mathrm{C}$ [17].

\section{Sports mechanism of drowning \\ Primary drowning}

This is by far the most common situation in aquatic sports, occurring by misjudgement of the ability to cope with the aquatic environment or falling into the water while participating in the sport.

\section{Secondary drowning}

This is classified as any injury/trauma or sudden illness acting as a precipitant factor producing the loss of consciousness or mentally or physically weakening the ability of the athlete to cope with the aquatic situation. The priority for the physician is to recognise a multiple disease situation in order to treat each one appropriately. Drowning in this category can be subdivided into three classes, as follows: 1) drowning caused by any injury/trauma involving a surfboard, boat crash or hang-gliding fall into the water, which acts as a precipitating factor; 2) drowning caused by any sudden illness such as cardiac disease, including myocardial ischaemia, arrhythmias, long-QT syndromes and hypertrophic cardiomyopathies or neurological disease, such as epilepsy, strokes and others; and 3) diving disease, which can be divided by the need or not to treat using a hyperbaric chamber.

\section{Diving disease requiring hyperbaric chamber treatment}

Decompression sickness (DCS) is a condition caused by the formation of nitrogen bubbles in a diver's blood and tissues during a rapid decompression ascent. Although they are generally quite tiny, these nitrogen bubbles can block blood flow to various parts of the body and may damage tissues irreversibly. DCS can produce many symptoms, and its effects may vary from joint pain and rashes to paralysis and death. Its potential severity has driven much research to prevent it and divers universally use dive tables or dive computers to limit their exposure and control their ascent speed. DCS is treated using hyperbaric oxygen therapy in a recompression chamber. If treated early, there is a significantly higher chance of successful recovery. This is an unusual cause of drowning, as the event usually takes place at the surface $[18,19]$.

Pulmonary over-pressurisation syndrome is a pulmonary trauma that may occur if the diver fails to expel air from the lungs during ascent. As the diver rises, the volume of the gas in the lung expands and can cause damage if the excess is not exhaled. Rupture of alveoli and a pneumothorax can occur. Air embolism is the most serious and feared consequence. While scuba diving, gas bubbles can enter the circulatory system through small ruptured veins in the lungs. In more severe cases these bubbles expand 
during ascent, following Boyle's law, and can pass through the heart to obstruct blood flow in the arteries of the brain or heart, known as air embolism. This occurs when a diver ascends rapidly without exhaling the air because of air shortage or panic. The diver may experience stroke, become unconscious or experience other nervous system complaints within minutes of surfacing. The brain is affected more than other organs because gas rises, and most divers are in a vertical position while ascending [19].

Diving disease not requiring hyperbaric chamber treatment

Compression barotrauma

Compression barotrauma usually occurs during descent, when the reduction in the volume of gas contained in the body is compressed by external pressure in a closed space that may not be compensated by some way out. This usually affects the sinuses and the middle ear, but can also affect the eyes (dive mask), bowels, teeth or skin. It may cause drowning if a rupture of the tympanic membrane causes disorientation, disturbing the diver's way out of the water. Barotrauma can also occur during ascent. A reverse squeeze occurs in the middle ear or sinus when a diver has an upper respiratory infection (a cold) and has used a nasal spray to open the breathing passages. As the spray wears off during diving, tissues swell and cause obstruction, resulting in a pressure difference and damage. During "bounce" diving the Eustachian tube may become inflamed and lead to a middle ear squeeze. Treatment involves using medication to open the Eustachian tube, anti-inflammatories and pain killers $[19,20]$.

\section{Narcosis}

Narcosis has been called the "rapture of the deep" and many divers compare narcosis to a feeling of pleasant drunkenness, as nitrogen narcosis is the most common form. The deeper a diver descends, the greater the narcosis. Although nitrogen is the principal component of air (79\%), other gases in a diver's tank, such as oxygen and carbon dioxide, are also narcotic at great depths. Diver narcosis is an altered state of mind caused by breathing nitrogen at a high partial pressure. It usually starts at depths of $\geqslant 50 \mathrm{~m}$, but the deeper the diver descends the higher will be the partial pressure of nitrogen and other gases in the air supply. In fact, divers sometimes use the "Martini rule" to roughly estimate the effects of narcosis during a dive. Depending upon the source, the Martini rule states that for every 10 or $20 \mathrm{~m}$ of depth, a diver experiences the narcotic effect of drinking one Martini. Narcosis affects a diver's coordination and decision-making ability, which may cause drowning. Another physical effect of narcosis is impaired thermoregulation. The shivering reaction that helps to warm a diver's body is reduced. A diver experiencing narcosis may be dangerously chilled, but typically feels warmer than is the case. This leads to the possibility of hypothermia. Physical impairment due to narcosis tends to begin at greater depths than the mental and emotional effects of narcosis. The recommended treatment is to reduce the diving pressure and, if oxygen is not involved directly in the narcosis, offer high-concentration oxygen at the surface $[19,20]$.

\section{Shallow water blackout}

Shallow water blackout, also known as dangerous underwater breath-holding behaviour (DUBB) [21], "breath-hold blackout" or "hypoxic blackout" is one of the most insidious forms of drowning and most often affects good or excellent swimmers and divers. In DUBB, swimmers, spear fishers, diving competitors or divers looking for sponges/pearls try to stay under water as long as possible. They will often take long, deep and fast breaths before submerging, trying to hyper-oxygenate to enable longer periods under the water. Hyper-oxygenation does not occur physiologically, instead using hyperventilation the swimmer exhales carbon dioxide, enabling a longer stay underwater [22] by blunting and delaying the normal respiratory drive to breathe (in normal, healthy individuals carbon dioxide accumulation is the primary respiratory drive). While submerged, hypocarbia delays the accumulation of carbon dioxide before the critical level that stimulates breathing is reached. Simultaneously, oxygen levels are falling and a critical level of hypoxia is reached where the swimmer become unconscious and drowns [21] if not rescued before water aspiration occurs. The sudden decrease in oxygen is more severe between $10 \mathrm{~m}$ and the surface. A recent report [21] has shown victims to be aged on average 17 years, mostly male, known to be advanced expert swimmers and engaged in intentional hyperventilation.

\section{Immersion pulmonary oedema}

Immersion pulmonary oedema (IPO) is characterised by the occurrence of dyspnoea, cough, haemoptysis and hypoxaemia during diving or strenuous surface swimming and may, on rare occasions, cause the loss of consciousness and cardiac arrest [23]. The pathophysiology is complex and only partly understood. Several factors are identified including: hydrostatic pressure gradient, cold-induced vasoconstriction, exertion, hydrostatic gradient between mouth and alveolus, inspiratory resistance by the regulator, increased gas density at depth or pre-dive hydration. The final common pathway is stress failure of the pulmonary capillaries leading to pulmonary capillary leak, interstitial loading, transudative oedema and eventually capillary rupture with migration of red blood cells and proteins to the alveolar space. It resembles 
high-altitude pulmonary oedema or even the effort of a drowning person to breathe with a water column in the main airways. Macroscopic differentiation between pulmonary oedema and drowning at autopsy is not possible. A survey of active divers [24] and triathletes [25] revealed symptoms suggestive of IPO in 1.1$1.4 \%$ of participants. In the majority of cases, symptoms resolved between $5 \mathrm{~min}$ and $24 \mathrm{~h}$. IPO can also cause panic, resulting in drowning or decompression illness. IPO is a diagnosis of exclusion, and water aspiration and not following decompression protocol during ascent must be excluded. IPO begins during diving and not afterwards. If IPO is not certain, hyperbaric oxygen treatment should be given. If IPO is diagnosed, treatment is similar to drowning grade 2 or 3 , depending on the extent of hypoxaemia.

\section{Ice-water sports causing drowning}

One variant of drowning that has attracted a lot of interest because of documented prolonged submersion times with good neurological outcomes is ice-water drowning. There have been well-documented submersions in excess of $1 \mathrm{~h}$, with subsequent good neurological recovery [26]. ORLOwsKI [26] recently reviewed the literature (from 1980 to 2015) on prolonged submersion accidents and the factors that influenced good neurological outcomes. A prolonged submersion was arbitrarily defined as $\geqslant 15 \mathrm{~min}$, and a good outcome as survival with mild or no neurological deficits. 38 cases had sufficient clinical details to permit analysis. Among those, $29(76 \%)$ cases were male and $25(66 \%)$ cases were aged $\leqslant 6$ years. 20 cases were documented to have occurred in ice-water (frozen surface). Of the remaining 18 cases, water temperature was not recorded in five cases, was $1-5^{\circ} \mathrm{C}$ in 10 cases and $5-7^{\circ} \mathrm{C}$ in one case; the warmest recorded water temperatures (two cases) were $9-10^{\circ} \mathrm{C}$. Core body temperature varied widely from $16.4^{\circ} \mathrm{C}$ to $35.7^{\circ} \mathrm{C}$ on admission, and did not correlate with water temperature, submersion time or estimated body surface area. Duration of resuscitation varied from $0-180 \mathrm{~min}$ until spontaneous heart rate and blood pressure were obtained. The longest documented submersion with good outcome was $66 \mathrm{~min}$ in a 2.5 -year-old girl, and required $>140$ min of resuscitation [27]. The longest estimated submersion time was $>83 \mathrm{~min}$ in a 7-year-old girl who required $64 \mathrm{~min}$ of CPR [28]. No pattern of optimal rewarming therapy emerged, although the most severe cases employed extra-corporeal membrane oxygenation. These accidents should be termed ice-water submersion, because $91 \%$ of the accidents with good outcomes occurred in water colder than $5^{\circ} \mathrm{C}$ and the longest submersions with good outcomes were the result of falling through ice. Core body temperature does not appear to influence outcome, and adds confusion because brain-dead victims are poikilothermic. Hypothermia may help protect the brain from anoxic-ischaemic injury, but does not appear to lessen the pulmonary injury, as all of the cases had acute respiratory distress syndrome (ARDS).

\section{Drowning chain of survival}

The "drowning chain of survival" [6] refers to a series of water safety interventions that, when put into action by laypeople or professionals, reduce the mortality associated with drowning (figure 1).

\section{Prevent drowning}

The most effective way to reduce the number of drowning deaths is prevention. It has been estimated that $80-90 \%$ of all drownings are preventable [11, 29]. Drowning prevention requires multiple layers of protection (table 2).

\section{Recognise distress and call for help}

The second element in the drowning chain is to recognise a person in distress in the water and know how to activate help [30]. A drowning risk assessment identifies a person at high risk of drowning by a near vertical body position, ineffective downward arm movements, ineffective pedalling or kicking leg actions and little or no forward progress in water. Sending someone to call for help upon recognising a person in water distress is a key element that ensures early activation of professional rescue service and emergency medical services [6].

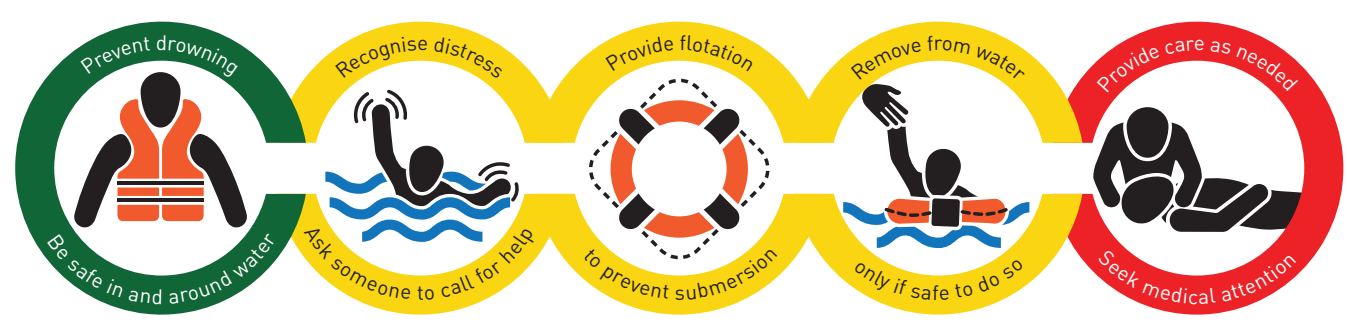

FIGURE 1 Drowning chain of survival. Reproduced from [6] with permission from the publisher. 


\section{TABLE 2 Preventive measures}

Watch children carefully, $84 \%$ of drownings occur because of bad adult supervision; the majority in or after the lunch hour. Begin swimming lessons from the age of 2 years, but be very careful at this time Avoid inflatable swimming aids such as "floaties"; they can give a false sense of security. Use a lifejacket! Never try to help rescue someone without being able to do it. Many people have died trying to do so Avoid drinking alcohol and eating before swimming

Don't dive in shallow water, there is a risk of cervical spine injury

\section{Beaches}

Always swim in a lifeguard-supervised area

Ask the lifeguard for safe places to swim or play

Read and follow warning signs posted on the beach

Do not overestimate your swimming capability; $46.6 \%$ of drowning victims thought they knew how to swim

Swim away from piers, rocks and stakes

Take lost children to the nearest lifeguard tower

$>80 \%$ of drownings occur in rip currents (the rip is usually a falsely calm deep place between two sand bars). If caught in a rip, swim transversally to the sand bar or let it take you away without fighting and wave for help

If you are fishing on rocks be cautious about waves that may sweep you into the ocean

Keep away from marine animals

Pools and similar

$>65 \%$ of deaths occur in fresh water, even on the coast

Fence off your pool and include a gate; approved fencing can decrease drowning by $50-70 \%$

Avoid toys around the pool; they are very attractive to children

Whenever infants or toddlers are in or around water, be within arm's length, providing

"touch supervision"

Turn off motor filters when using the pool

Always use portable phones in pool areas, so you are not called away to answer

Don't attempt hyperventilation to increase submersion time

Use warning sign to indicate shallow water

Learn how to react to a drowning situation, $>42 \%$ of pools owner are not aware of first-aid techniques; be careful!

\section{Provide flotation to stop the process of drowning}

The next priority is to interrupt the drowning process by providing flotation to the victim as an interim measure to reduce the risk of airways submersion [6]. This buys valuable time for those at the scene to initiate rescue efforts and for emergency services to arrive. It is critical that laypeople take precautions to avoid becoming another victim by engaging in inappropriate or dangerous rescue responses [5, 15]. Reaching out with, throwing or dropping the buoyancy aid without entering the water is the preferred method of providing flotation to a drowning victim [31]. Because many victims cling to their would-be rescuer, it is better to always approach a struggling victim with an intermediary object.

\section{In-water resuscitation}

If not interrupted, the drowning process leads first to unconsciousness and apnoea, followed by cardiac arrest within minutes. During this short window of opportunity, immediate in-water ventilation may benefit the victim if provided safely and effectively [32]. For unconscious victims, in-water resuscitation can increase discharge from hospital without sequelae by more than three-fold. In-water ventilation is only possible if the rescuer is highly trained. Chest compression while the rescuer and victim are in deep water is futile, so assessment for a pulse does not serve any purpose. Victims with respiratory arrest only usually respond after a few rescue breaths. If there is no response, the victim should be assumed to be in cardiac arrest and be rescued as quickly as possible to dry land, where effective CPR can be initiated.

\section{Cervical spine injury}

Very few studies are undertaken of how often in-water cervical spine injury occurs [33-35]. One of these, concerning sand beaches, retrospectively evaluated 46060 water rescues and demonstrated that the incidence is very low $(0.009 \%)$ [34]. In another study with $>2400$ drownings, only $11(<0.5 \%)$ had a cervical spine injury and all of these had a history of obvious trauma [33]. Other water locations may have different rates depending on a wide variety of elements. Furthermore, any time spent on immobilising the cervical spine in unconscious victims with no signs of trauma could lead to a cardiopulmonary deterioration and even death. Considering the low incidence of cervical spine injury and the high risk of 
wasted time when ventilation is needed, routine cervical spine immobilisation of water rescue victims, without reference to whether a traumatic injury was sustained, is not recommended [36, 37].

\section{Remove from water: rescue only if safe to do so}

It is fundamental that laypeople take precautions not to become victims themselves by engaging in inappropriate or dangerous rescue responses. The lay rescuer may first consider helping the victim by throwing, reaching or wading to the victim [6]. To enter the water is a personal decision and may depend on a personal relationship with the victim (for example parents and children), depth of the water, distance to swim and swimming skills. In order to mitigate the risk to the bystander rescuer it is recommended that a flotation device or connecting rope is used, although these may increase the risk [31]. Removing the victim from the water is essential in order to end the drowning process [32] and provides a better setting for assessment and care of the victim.

Preferably, a drowning victim should be transported out of the water in as near to a horizontal position as possible, but with the head still maintained above body level. The airways must be kept open at all times if possible [38].

\section{Provide care as needed: from basic life support to hospital treatment}

Basic life support

Once in a dry place, the victim should be placed supine with the trunk and head at the same level. Perform the standard checks for responsiveness and breathing [32]. If the victim is unconscious but breathing, the recovery position should be used [38]. If the victim is not breathing, ventilation is essential [1, 4, 37]. If the victim has a cardiac arrest from drowning, it should be understood that this is primarily due to lack of oxygen $[4,15,37,39]$. For this reason, it is important that CPR follows the airway-breathing-circulation sequence $[39,40]$. Upper airway management is always challenging due to vomiting and the pulmonary oedema fluid that interferes with airway management, while at the same time pulmonary injury makes the initial ventilation difficult because of decreased pulmonary compliance [37, 41]. This means five initial breaths followed by 30 chest compressions, continuing with two breaths to 30 compressions until signs of life reappear, rescuer exhaustion occurs or advance life support becomes available. Because cardiac arrest in a drowning victim is caused by asphyxia, cardiac compression only CPR is not the best approach [37, 39].

The most frequent complication during resuscitation of a drowning victim is regurgitation of stomach contents (65-86\%) [42]. The presence of vomitus in the airway can result in further aspiration injury and impairment of oxygenation [20]. Active efforts to expel water from the airway (abdominal thrusts or placing the victim head down) should be avoided, as they only delay the initiation of ventilation, increase the risk of vomiting by more than five-fold, and thereby lead to a significant increase in mortality [32, 38].

A study has shown that $<10 \%$ of all drowning victims are in ventricular fibrillation [43], so the effectiveness of an automated external defibrillator is low if the drowning is not precipitated by a cardiac cause.

\section{Advanced life support}

Early advanced life support will contribute to a good drowning outcome and should be initiated as soon as possible [1]. Cardiopulmonary or isolated respiratory arrest comprises only $\sim 0.5 \%$ of all rescues. In this situation it is clear that resuscitation has to be started. For other, less serious situations, a classification system has been developed [4] and revalidated [44] to assist lifeguards, ambulance personnel and physicians in treatment. This classification (figure 2) [1], stratified into six grades plus a rescue and a non-resuscitation condition, encompassing all the support from the site of the accident to the hospital, recommends the most appropriate treatment, and shows the likelihood of death based on the severity of injury [4, 44]. To save time, medical equipment should be brought to the victim instead of the victim to the ambulance. Recommendations for when to start and stop resuscitation are different to non-drowning-related cardiac arrest (table 3) $[1,13]$.

At grade 6 [4], resuscitation started by a layperson or lifeguard at the scene must be continued by the emergency medical services; the first priority should be adequate oxygenation and ventilation using a face mask with supplemental oxygen or bag-mask ventilation with $15 \mathrm{~L}$ of oxygen until an orotracheal tube can be inserted. Meanwhile, cardiac compression should be continued. Once intubated, most victims can be oxygenated and ventilated effectively even in situations where copious pulmonary oedema fluid fills the entire endotracheal tube. Aspirate the orotracheal tube only when fluid makes effective ventilation impossible. Suctioning can disturb oxygenation and should be balanced against the need to ventilate and oxygenate. For cardiac monitoring, the presenting rhythm in cases of cardiac arrest following drowning is usually asystole. Ventricular fibrillation is rarely reported, but may occur if there is a history of coronary artery disease, due to the use of adrenaline or in the presence of severe hypothermia [13]. If ventricular fibrillation is present, defibrillation should be attempted. Peripheral venous access is the preferred route for drug administration in the pre-hospital setting. Endotracheal administration of drugs is not recommended 


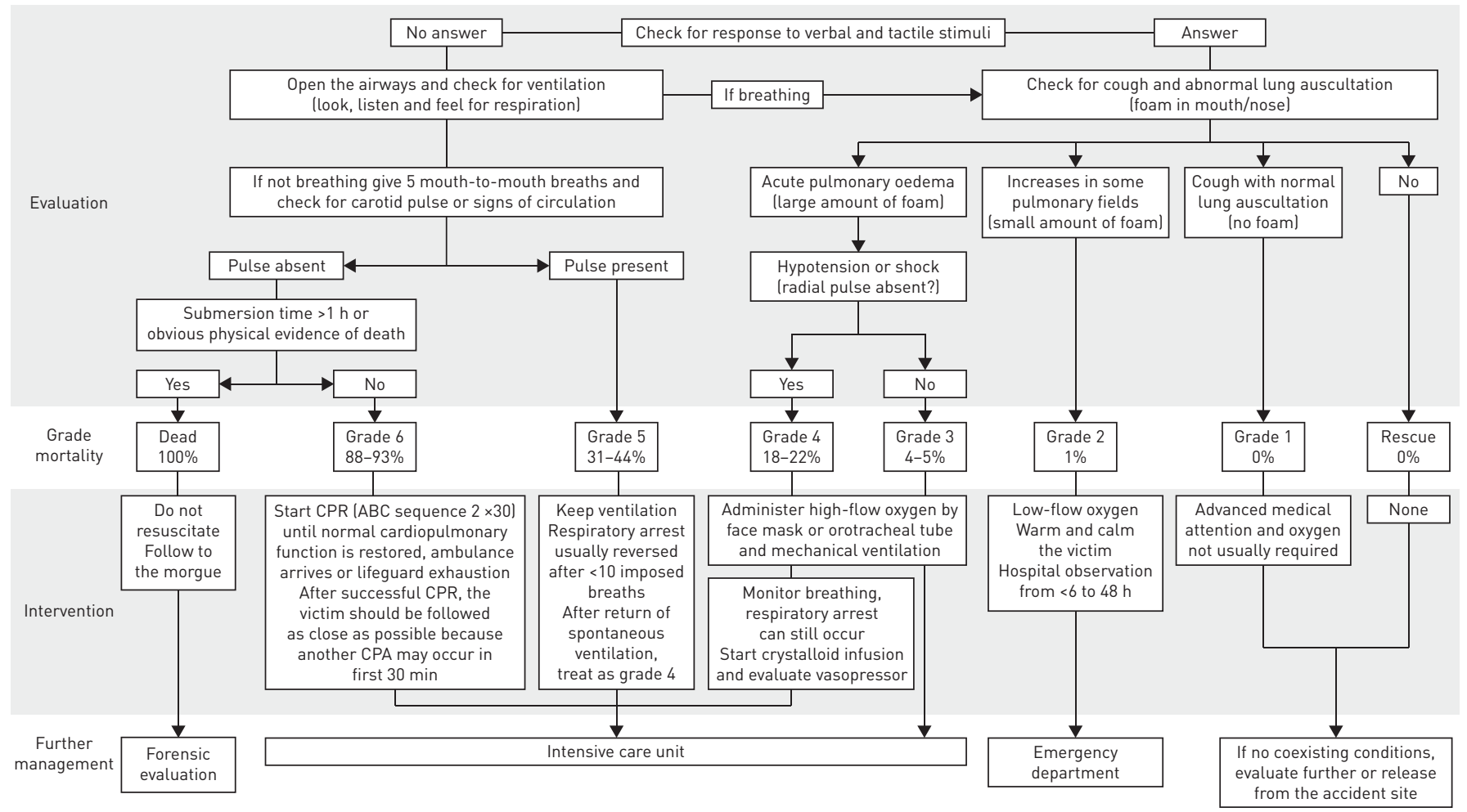

FIGURE 2 Drowning severity classification and flow chart strategy decision based on evaluation of 87339 rescues [1, 4, 30]. CPR: cardiopulmonary resuscitation; ABC: airway, breathing, compression; CPA: cardiopulmonary arrest. Reproduced from [1], with permission.

for drowning [39]. Cumulative doses of adrenaline $1 \mathrm{mg}$ i.v. (or $0.01 \mathrm{mg} \cdot \mathrm{kg}^{-1}$ ) can be considered. Once the resuscitation setting is organised, an orogastric tube can be placed to reduce gastric distention and to prevent further aspiration. This is indicated if abdominal distention restricts ventilation. If advanced life support resuscitation is not successful after $15 \mathrm{~min}$ in a hypothermic victim, consider transport to a hospital where advanced warming measures can be accomplished while resuscitation is continued during transport. Grade 5 cases [4] are usually reversed by mouth-to-mouth ventilation by bystanders or lifeguards by the time advanced life support arrives at the scene. If there is spontaneous ventilation, the protocol for grade 4 is followed. A grade 4 [4] patient may initially be able to maintain adequate oxygenation through an abnormally high respiratory rate. The objective of oxygenation is to achieve a pre-hospital peripheral saturation $>92 \%$ by administering oxygen via face mask at a rate of $15 \mathrm{~L} \cdot \mathrm{min}^{-1}$. Early intubation and mechanical ventilation are indicated as soon as possible, because of respiratory fatigue, even when good oxygenation is achieved using the face mask. Once intubated, most victims can be oxygenated and ventilated effectively. Patients should be sedated to tolerate intubation and artificial mechanical ventilation providing tidal volume of $\geqslant 5 \mathrm{~mL} \cdot \mathrm{kg}^{-1}$ of body weight. Inspiratory oxygen fraction $\left(\mathrm{FIO}_{2}\right)$ can start at 1.0 , but should be reduced when possible. If hypotension is not corrected by oxygen, a rapid crystalloid infusion

TABLE 3 Drowning: when to initiate cardiopulmonary resuscitation (CPR) and when to discontinue

\section{Recommendations}

When to begin?

Give ventilatory support for respiratory distress/arrest to avoid cardiac arrest Start CPR in all submersions lasting $<60$ min that do not present obvious physical evidence of death (rigor mortis, body decomposition or dependent lividityl

When to discontinue? Basic life support should continue until signs of life reappear, rescuer exhaustion or advanced life support takes over

Advanced life support should be ongoing until the patient has been rewarmed (if hypothermic) or asystole persists for $>20 \mathrm{~min}$

Reproduced from [1], with permission. 
TABLE 4 Who needs further medical help after rescue from the water?

\section{Send to hospital}

Any patient who lost consciousness, even for a brief period

Any patient who required expired air resuscitation (rescue breathing)

Any patient who required cardiopulmonary resuscitation

Any patient in whom a serious condition is suspected, such as heart attack, spinal injury, other injury, asthma, epilepsy, jellyfish stings, intoxication, delirium, etc.

\section{Consider for release from care at the scene}

If, after 10-15 min of careful observation, while being warmed with blankets or other coverings as required, the patient has $A L L$ of the following:

No cough

Normal rate of breathing

Normal circulation as measured by pulse strength and rate and blood pressure (if available)

Normal colour and skin perfusion

No shivering

Fully conscious, awake and alert

In such cases, it is unwise for the patient to drive a vehicle and the patient should be so advised

If any of these conditions do not apply or if the lifesaver has any doubt, then the patient should be advised to seek early medical attention

\section{There is always a risk of delayed lung complications}

All immersion victims should therefore be warned that if they later develop cough, breathlessness, fever or any other worrying symptom, they should seek medical advice immediately. It is preferable that these cases do not return to a home environment where they are alone for the next $24 \mathrm{~h}$

should be used [16]. In grade 3 cases the issue is to decide whether there would be a greater benefit from invasive ventilation than using face mask oxygenation. Only $27.6 \%$ of these victims tolerate noninvasive ventilatory support alone [4]. Grade 2 cases usually only require oxygen by nasal cannula (93.2\%). In grade 1 and rescue cases advanced medical attention and oxygen are not usually required.

As the majority of drowning cases have only mild distress or may not actually aspirate water it is important for responders to know when to call emergency medical services (ambulance) or seek medical assistance/ hospital care (table 4). Emergency department attendance is recommended for all grade 2-6 patients.

\section{Hospital treatment}

Decisions in the emergency department about admission to an intensive care unit (ICU) or hospital bed versus observation in an emergency department or discharge home should include a thorough history of the incident and previous illness, physical examination and diagnostic studies including chest radiography and arterial blood gas measurement. In addition, electrolytes, blood urea nitrogen, creatinine and haemoglobin should be assessed, although perturbations in these laboratory tests are unusual. Patients graded 3-6 should be admitted to an ICU for close observation and therapy. Patients graded 2 can be observed in the emergency room for 6-24 h, but grade 1 and rescue cases with no complaints or associated illness or trauma can be released home [1].

That drowning may have been precipitated by an injury or medical condition such as trauma, seizure or cardiac arrhythmia should be considered [4], as this might guide specific treatment.

Respiratory system

Patients graded 4-6 will usually arrive at the hospital on mechanical ventilation with acceptable oxygenation. If not, the emergency department physician should follow grade 4 ventilation protocols. Positive end-expiratory pressure (PEEP) should be added initially at a level of $5 \mathrm{cmH}_{2} \mathrm{O}$ and then increased by $2-3 \mathrm{cmH}_{2} \mathrm{O}$ increments if possible until the desired intrapulmonary shunt (QS:QT) of $\leqslant 20 \%$, or $\mathrm{PaO}_{2}: \mathrm{FIO}_{2}$ of $\geqslant 250$ is achieved. Once the desired oxygenation is achieved, PEEP level should be maintained unchanged for $48 \mathrm{~h}$. This period is needed to permit adequate surfactant regeneration. It is suggested that an early weaning from the ventilator may cause the return of pulmonary oedema with the need for re-intubation, a prolonged hospital stay and further morbidity [1]. A clinical picture very similar to ARDS is common after significant drowning episodes (grades 3-6). Management is similar to that of other patients with ARDS. Lung-protective ventilation involving permissive hypercapnia is probably not suitable for grade 6 drowning victims with significant hypoxic-ischaemic brain injury. In selected cases, continuous positive airway pressure (CPAP) may be provided by mask only in cooperative adolescents, or by nasal cannula in infants who are obligate nasal breathers, but this is not usually tolerated. If pulmonary and psychological status allows the patient to breathe without fighting, CPAP or pressure-support 
ventilation can be a good choice. The clinician must be aware of and constantly vigilant for volutrauma and barotrauma during the ventilation of drowning victims [45]. A spontaneous pneumothorax is common (10\%) secondary to positive-pressure ventilation and local areas of hyperinflation.

Pools, rivers and beaches generally have insufficient bacteria colonisation to promote pneumonia in the immediate post-drowning period [45]. Pneumonia is often misdiagnosed initially because of the early radiographic appearance of water in the lungs [46]. If the patient needs mechanical respiratory assistance, the incidence of ventilator-associated pneumonia increases to $34-52 \%$ on the third or fourth day of hospitalisation, when pulmonary oedema is resolving [46]. Prophylactic antibiotics tend to only select out more resistant and more aggressive organisms [47]. It is advised to monitor tracheal aspirates daily using Gram stain, culture and sensitivity. At the first sign of pulmonary infection, usually around the first $48-72 \mathrm{~h}$ (as gauged by prolonged fever, sustained leukocytosis, persistent or new pulmonary infiltrates and leukocyte response in the tracheal aspirate), antibiotic therapy can be initiated on the basis of predominant organism growth in the water where the drowning occurred. If there are reasons to suspect ventilator-associated pneumonia, antibiotic therapy should be directed at the sensitivity of the predominant micro-organisms at the ICU, or by culture if available. Fibre-optic bronchoscopy may be useful for evaluation of infection. Corticosteroids should not be used for pulmonary injury, except for bronchospasm [1].

\section{Circulatory system}

Cardiac dysfunction with low cardiac output is common immediately after severe drowning [12]. This may cause a low blood pressure and also adds a cardiogenic component to the noncardiogenic pulmonary oedema. The low blood pressure can be corrected using better oxygenation, rapid crystalloid infusion and restoration of normal body temperature. Vasopressor infusion should only be used in refractory hypotension. A basic measurement is to monitor urine output. Echocardiography to assess cardiac function and ejection fractions can help to guide the clinician in titrating inotropic agents, vasopressors or both if volume crystalloid replacement has failed [1]. When pulmonary oedema occurs after drowning, there is no evidence to support the use of any specific fluid therapy for salt and fresh water drowning [12], the use of diuretics or water restriction.

Metabolic acidosis occurs in 70\% of patients arriving at hospital after a drowning episode [4]. The acidosis should be corrected when $\mathrm{pH}$ is $<7.2$ or the bicarbonate is $<12 \mathrm{mEq} \cdot \mathrm{L}^{-1}$ in spite of adequate ventilatory support. Significant depletion of bicarbonate is rarely present in the first $10-15 \mathrm{~min}$ of CPR, and its use is not indicated in the initial resuscitation period. In hypothermic patients, arterial blood gases do not need to be temperature corrected (the $\alpha$-stat or $\mathrm{pH}$-stat concept) [48].

\section{Neurological system}

The most important complication in grade 6 patients after a drowning episode, beyond reversible pulmonary injury, is anoxic-ischaemic cerebral insult. Most late deaths and long-term sequelae of drowning are neurological in origin [45], so every effort in the early stages after rescue should be directed at resuscitating the brain and preventing further neurological damage. These steps include providing adequate oxygenation and cerebral perfusion. Any victim who remains comatose and unresponsive after successful CPR or deteriorates neurologically should undergo frequent neurological function assessment for the development of cerebral oedema, and should be treated accordingly [49]. Drowning victims with restoration of adequate spontaneous circulation who remain comatose should not be actively rewarmed to temperature values $>32-34^{\circ} \mathrm{C}$. If core temperature exceeds $34^{\circ} \mathrm{C}$, therapeutic hypothermia $\left(32-34^{\circ} \mathrm{C}\right)$ should be achieved as soon as possible and sustained for $12-24 \mathrm{~h}$. Although there is insufficient evidence to support a specific target arterial carbon dioxide tension or oxygen saturation during and after resuscitation, hypoxaemia should be avoided. More research is needed to evaluate specific efficacy of neuroresuscitative therapies in drowning victims [1].

\section{Unusual complications}

Important medical complications after drowning are rare and are almost all restricted to grade 6 cases. Rarely, drowning victims who have normal chest radiography at initial assessment in the emergency department develop fulminant pulmonary oedema as long as $12 \mathrm{~h}$ after the incident. Whether this late-onset pulmonary oedema is ARDS, neurogenic pulmonary oedema, IPO or just an airway hyperreactive to water aspiration is unclear. Renal insufficiency or renal failure is rare in drowning victims, but can occur secondary to anoxia, shock or haemoglobinuria [1].

\section{Outcome}

With the progression of intensive care therapy, prognostication is increasingly based on neurological outcome [15]. Of drowning cases graded 1-5, 95\% return home without sequelae [4]. 
In grade 6 patients, prognostication is important when counselling family members of drowning victims in the early stages after the incident and in deciding which patients are likely to have a good outcome with standard supportive therapy and which should be candidates for intensive cerebral resuscitation therapies [15].

Victims who remain comatose or deteriorate neurologically should undergo intensive assessment and care $[1,49]$. Not only is successful resuscitation following a long submersion in cold or icy water possible, some anecdotal warm water cases have been described to survive without sequelae $[4,14,50]$. Multiple studies $[1,4,9,13-15,17,29,32,50,51]$ have established that outcome is almost solely determined by a single factor: duration of submersion.

Patients who are improving but remain unresponsive have a 50\% likelihood of a good outcome. Most patients who are definitely improving and are alert or are stuporous or obtunded but respond to stimuli 2-6 h after the incident have normal or near-normal neurological outcomes.

\section{References}

Szpilman D, Bierens JJLM, Handley AJ, et al. Drowning. N Engl J Med 2012; 366: 2102-2110.

2 World Health Organization. Global Report on Drowning: Preventing a Leading Killer. Geneva, World Health Organization, 2014.

3 Lu TH, Lunetta P, Walker S. Quality of cause-of-death reporting using ICD-10 drowning codes: a descriptive study of 69 countries. BMC Med Res Methodol 2010; 10: 30.

4 Szpilman D. Near-drowning and drowning classification: a proposal to stratify mortality based on the analysis of 1831 cases. Chest 1997; 112: 660-665.

5 Venema AM, Groothoff JW, Bierens JJ. The role of bystanders during rescue and resuscitation of drowning victims. Resuscitation 2010; 81: 434-439.

6 Szpilman D, Webber J, Quan L, et al. Creating a drowning chain of survival. Resuscitation 2014; 85: 1149-1152.

7 Mitchell RJ, Williamson AM, Olivier J. Estimates of drowning morbidity and mortality adjusted for exposure to risk. Inj Prev 2010; 16: 261-266.

8 Van Beeck EF, Branche CM, Szpilman D, et al. A new definition of drowning: towards documentation and prevention of a global public health problem. Bull World Health Organ 2005; 83: 853-856.

9 Idris AH, Berg RA, Bierens JJLM, et al. Recommended guidelines for uniform reporting of data from drowning: the "Utstein style". Circulation 2003; 108: 2565-2574.

10 Szpilman D, Elmann J, Cruz-Filho FES. Dry-drowning - Fact or Myth? Poster presentation, World Congress on Drowning, The Netherlands, 2002, Book of Abstracts; p. 176.

11 Lunetta P, Modell JH, Sajantila A. What is the incidence and significance of "dry-lungs" in bodies found in water? Am J Forensic Med Pathol 2004; 25: 291-301.

12 Orlowski JP, Abulleil MM, Phillips JM. The hemodynamic and cardiovascular effects of near-drowning in hypotonic, isotonic, or hypertonic solutions. Ann Emerg Med 1989; 18: 1044-1049.

13 Grmec S, Strnad M, Podgorsek D. Comparison of the characteristics and outcome among patients suffering from out-of-hospital primary cardiac arrest and drowning victims in cardiac arrest. Int J Emerg Med 2009; 2: 7-12.

14 Tipton MJ, Golden FS. A proposed decision-making guide for the search, rescue and resuscitation of submersion (head under) victims based on expert opinion. Resuscitation 2011; 82: 819-824.

15 Orlowski JP, Szpilman D. Drowning. Rescue, resuscitation, and reanimation. Pediatr Clin North Am 2001; 48: 627-646.

16 Modell JH, Moya F, Newby EJ, et al. The effects of fluid volume in seawater drowning. Ann Intern Med 1967; 67: 68-80.

17 Polderman KH. Application of therapeutic hypothermia in the ICU: opportunities and pitfalls of a promising treatment modality. Part 1: indications and evidence. Intensive Care Med 2004; 30: 556-575.

18 Vann RD, Butler FK, Mitchell SJ, et al. Decompression illness. Lancet 2011; 377: 153-164.

19 Spira A. Diving and marine medicine review part 11: diving diseases. J Travel Med 1999; 6: 180-198.

20 Denoble PJ, Caruso JL, Dear Gde L, et al. Common causes of open-circuit recreational diving fatalities. Undersea Hyperb Med 2008; 35: 393-406.

21 Boyd C, Levy A, McProud T, et al. Fatal and nonfatal drowning outcomes related to dangerous underwater breath-holding behaviors - New York State, 1988-2011. MMWR Morb Mortal Wkly Rep 2015; 64: 518-521.

22 Craig AB Jr. Summary of 58 cases of loss of consciousness during underwater swimming and diving. Med Sci Sports 1976; 8: 171-175.

23 Cochard G, Arvieux J, Lacour JM, et al. Pulmonary edema in scuba divers: recurrence and fatal outcome. Undersea Hyperb Med 2005; 32: 39-44.

24 Pons M, Blickenstorfer D, Oechslin E. Pulmonary oedema in healthy persons during scuba-diving and swimming. Eur Respir J 1995; 8: 762-767.

25 Miller C 3rd, Calder-Becker K, Modave F. Swimming-induced pulmonary edema in triathletes. Am J Emerg Med 2010; 28: 941-946.

26 Orlowski JP. Drowning, near-drowning, and ice-water drowning. JAMA 1988; 260: 390-391.

27 Bolte RG, Black PG, Bowers RS, et al. The use of extracorporeal rewarming in a child submerged for 66 minutes. JAMA 1988; 260: 377-379.

28 Romlin BS, Winberg $\mathrm{H}$, Janson $\mathrm{M}$, et al. Excellent outcome with extracorporeal membrane oxygenation after accidental profound hypothermia $\left(13.8^{\circ} \mathrm{C}\right)$ and drowning. Crit Care Med 2015; 43: e521-e525.

29 Quan L, Pilkey D, Gomez A, et al. Analysis of paediatric drowning deaths in Washington State using the child death review (CDR) for surveillance: what CDR does and does not tell us about lethal drowning injury. Inj Prev 2011; 17: Suppl. 1, i28-i33.

30 Langendorfer SJ. Applying a development perspective to aquatics and swimming. In: Kjendlie PL, Stallman RK, Cabri J, eds. Biomechanics and Medicine in Swimming. 11th Edn. 2010; pp. 20-22. 
31 Turgut A, Turgut T. A study on rescuer drowning and multiple drowning incidents. J Safety Res 2012; 43: 129-132.

32 Szpilman D, Soares M. In-water resuscitation - is it worthwhile? Resuscitation 2004; 63: 25-31.

33 Watson RS, Cummings P, Quan L, et al. Cervical spine injuries among submersion victims. J Trauma 2001; 51: 658-662.

34 Szpilman D, Brewster C, Cruz-Filho FES. Aquatic Cervical Spine Injury - How Often do We Have to Worry? Oral presentation, World Congress on Drowning, The Netherlands 2002.

35 Szpilman D. Aquatic Cervical and Head Trauma: Nobody Told Me it Could be a Jump in the Darkness! World Conference on Drowning Prevention, Danang, Vietnam 2011, Book of Abstracts, ISBN: 978-0-909689-33-9, p. 153.

36 Wernicke P, Szpilman D. Immobilization and extraction of spinal injuries. In: Bierens J, ed. Drowning: Prevention, Rescue, Treatment. Berlin, Springer-Verlag, 2014; 621-628.

37 Soar J, Perkins GD, Abbas G, et al. European Resuscitation Council Guidelines for Resuscitation 2010 Section 8. Cardiac arrest in special circumstances: electrolyte abnormalities, poisoning, drowning, accidental hypothermia, hyperthermia, asthma, anaphylaxis, cardiac surgery, trauma, pregnancy, electrocution. Resuscitation 2010; 81: 1400-1433.

38 Szpilman D, Handley A. Positioning the drowning victim. In: Bierens J, ed. Drowning: Prevention, Rescue, Treatment. Berlin, Springer-Verlag, 2006; pp. 336-341.

39 Vanden Hoek TL, Morrison LJ, Shuster M, et al. Part 12: cardiac arrest in special situations: drowning. 2010 American Heart Association guidelines for cardiopulmonary resuscitation and emergency cardiovascular care. Circulation 2010; 122: S829-S861.

40 Kitamura $\mathrm{T}$, Iwami $\mathrm{T}$, Kawamura $\mathrm{T}$, et al. Conventional and chest-compression-only cardiopulmonary resuscitation by bystanders for children who have out-of-hospital cardiac arrests: a prospective, nationwide, population-based cohort study. Lancet 2010; 375: 1347-1354.

41 Baker PA, Webber JB. Failure to ventilate with supraglottic airways after drowning. Anaesth Intensive Care 2011; 39: 675-677.

42 Manolios N, Mackie I. Drowning and near-drowning on Australian beaches patrolled by life-savers: a 10-year study, 1973-1983. Med J Aust 1988; 148: 165-167.

43 Beerman S, Lofgren B. Automated external defibrillator in the aquatic environment. In: Bierens J, ed. Drowning: Prevention, Rescue, Treatment. Berlin, Springer-Verlag, 2006; pp. 331-336.

44 Szpilman D, Elmann J, Cruz-Filho RES. Drowning classification: a revalidation study based on the analysis of 930 cases over 10 years. World Congress on Drowning, Amsterdam 2002, Book of Abstracts, p. 66.

45 Orlowski JP. Drowning, near-drowning, and ice-water submersion. Pediatr Clin North Am 1987; 34: 75-92.

46 van Berkel M, Bierens JJ, Lie RL, et al. Pulmonary oedema, pneumonia and mortality in submersions victims. A Retrospective study in 125 patients. Intensive Care Med 1996; 22: 101-107.

47 Wood C. Towards evidence based emergency medicine: best BETs from the Manchester Royal Infirmary. BET 1: prophylactic antibiotics in near-drowning. Emerg Med J 2010; 27: 393-394.

48 Abdul Aziz KA, Meduoye A. Is pH-stat or alpha-stat the best technique to follow in patients undergoing deep hypothermic circulatory arrest? Interact Cardiovasc Thorac Surg 2010; 10: 271-282.

49 The NICE-SUGAR Study Investigators. Intensive versus conventional glucose control in critically ill patients. N Engl J Med 2009; 360: 1283-1297.

50 Szpilman D. A case report of 22-minute submersion in warm water without sequelae. In: Bierens JJLM, ed. Drowning: Prevention, Rescue, Treatment. Berlin, Springer-Verlag, 2005; pp. 375-376.

51 Warner D, Knape J. Recommendations and consensus: brain resuscitation in the drowning victim. In: Bierens JJLM, ed. Drowning: Prevention, Rescue, Treatment. Berlin, SpringerVerlag, 2006; pp. 436-439. 